\title{
Evaluation of proximity of the floor of the maxillary sinus to the alveolar bone crest, using digital panoramic imaging system, in Erbil city \\ Received: $10 / 2 / 2011$ \\ Accepted: 13/9/2011
}

\begin{abstract}
Sarkawt H. Ali* $\quad$ Saeed Nadhim** $\quad$ Othman A. Omar***

\begin{tabular}{ccc}
\hline Sarkawt H. Ali* & Saeed Nadhim** & Othman A. Omar*** \\
\hline Abstract
\end{tabular}

Background and objectives: measuring the distance between the lowest point of the maxillary sinus floor and maxillary alveolar bone crest seen in the digital panoramic images and the effect of alveolar bone resorption to evaluate the clinical benefit of using software measurement program to measure the height of the alveolar bone in the region of maxillary sinus.
\end{abstract}

Method: Digital panoramic images were analyzed using the software computer programs that existed in the system to measure the distance from the lowest point of the maxillary sinus floor and maxillary alveolar bone crest. Images were divided into four groups (1st group dentulous cases, 2nd group single dental extraction, 3rd group multiple dental extraction, 4th group edentulous cases) and each group consist of 50 panoramic images.

Results: The mean distance of sinus floor to bone crest in fourth group cases was approximately half of the distance in first group due to extraction of teeth and there was highly significant difference $(P<0.001)$ between first group, third group and fourth group images, and there was significant difference $(P<0.005)$ between first group and second group images.

Conclusion: It was concluded that the objective assessment of alveolar bone height and its 2-dimensional relation to maxillary teeth was greatly helpful by applying the software program.

Keywords: Panoramic radiograph, deepest point of floor of maxillary sinus, crest of alveolar bone.

\section{Introduction}

The maxillary sinus (antrum of Highmore) is the first of the paranasal sinuses to develop and ends its growth at approximately 20 years of age with the eruption of the maxillary third molars. ${ }^{1}$ The maxillary sinuses are air-containing spaces that occupy the maxillary bone bilaterally. It is described as a four-sided pyramid, with the base lying vertically on the medial surface and forming the lateral nasal wall. Anteriorly and laterally sinus extends to the region of the bicuspid or cuspid teeth. The floor of the sinus forms the base of the alveolar process. ${ }^{2,3}$ Radiographic examination of the maxillary sinus may be accomplished with a variety of exposure including periapical, occlusal and panoramic views, which will in most instances provide adequate information to either confirm or rule out any pathologic lesions of the sinus. ${ }^{4,5}$ Panoramic radiography is an extraoral radiographic technique that is used to examine the upper and lower jaws on a single film. Panoramic radiography is a diagnostic tool in locating foreign bodies within the sinus particularly roots of maxillary posterior teeth or osseous fragments that have been displaced by trauma or during tooth removal. Dental pathologic condition such as cysts or granulomas may produce radiolucent lesions that extend into the sinus cavity. ${ }^{6,7,8}$ Knowledge of the relationship between the root apex and the inferior wall of the maxillary sinus are crucial

*Department of Oral Diagnosis, College Dentistry, Hawler Medical University, Erbil-Iraq..

$* *$ Department of Surgery, College of Medicine, Hawler Medical University, Erbil-Iraq.

***Department of Maxillofacial Surgery, College Dentistry, Hawler Medical University, Erbil-Iraq. 
for diagnosing and treating a sinus pathology as well as in assisting in dental implantation. Accordingly, knowledge of the topography between the root apex and the inferior wall of the maxillary sinus is important for diagnosing and planning dental implantation, endodontic procedures, and orthodontic treatment. ${ }^{9-11}$

\section{Aims of the study}

1. To measure the distance from the deepest point of the maxillary sinus floor to maxillary posterior teeth.

2. To provide, dentists, oral and maxillofacial surgeons with the above basic information in our population to avoid complications during surgical procedures in the area of upper posterior teeth.

3. To know the effect of dental extraction on alveolar bone resorption.

4. To evaluate the clinical benefit of using software measurement program to measure the height of the alveolar bone in the region of maxillary sinus.

\section{Method}

The sample: A retrospective cross sectional study of Orthopantomogram (OPG) during 5 years for patients attended the Department of Radiology of specialized dental polyclinic in Erbil city, from 2005 to 2010 for those patients who took OPGs from the mentioned hospital and .The sample size (OPG cases) divided in to 4 groups and each group consists of (50) Orthopantomograms.

1. The first group completely dentulous (control group) (CG).

2. The second group single dental extraction (single dental extraction must be in the region of maxillary sinus) (SDE).

3. The third group multiple dental extraction ( multiple dental extractions must be in the region of maxillary sinus) (MDE).

4. The fourth group were completely edentulous (CE).

Methods: The software program that existed in the digital computerized machine for measurement of any length, angle or any distance between two points, lines or more on the panoramic radiograph. It was used this software program for measuring a straight line from the deepest point of the maxillary sinus floor to the nearest alveolar bone crest, this line will be measured by $\mathrm{mm}$ and all calibrations will be done for saving the images, to make a comparison between groups(group1, group2, group3, group4) and the software was developed at the Research Services Branch (RSB) of the National Institute of Mental Health (NIMH) Scion Image can be used to acquire, display, edit, enhance, analyze, and animate images. It supports many standard image processing functions including contrast enhancement, density profiling, smoothing, sharpening, edge detection, median filtering, and spatial convolution with user defined kernels the software can be used to measure area, mean, and centroid of an area within a perimeter of a user defined region of interest. It provides tools for measuring path lengths and angles.

Statistical Analysis: Data analysis carried out using SPSS program Statistical package of social sciences version 15.0 (SPSS Inc., Chicago, IL, USA). Statistical analysis includes both descriptive and inferential statistics for analyzing the data obtained from the study to explain the results.

1. Descriptive statistics; which include tables with percentages and graphical presentation to clarify the results.

2. Inferential statistics; these were used to accept or reject hypothesis and this include Chi-square test, t-test and ANOVA.

\section{Results}

Calibration of SF-BC distances between four groups: Table (1) demonstrates the mean and standard deviation of SF-BC values for each groups. The mean and standard deviation SF-BC distances of the first group (CG) was $9.19,1.47$ Figure(1), the mean and standard deviation SF-BC distances of the second group (SDE) was $7.13,1.81$ Figure (2), the mean and standard deviation SF-BC distances of the third group (MDE) was 5.9, 1.45 Figure (3) and , the mean and standard deviation SF-BC distances of the fourth group (CE) was 4.74, 1.18 Figure (4), and $P$ value less than 
$(P<0.001)$ there were highly significance in SF-BC distances measurement among the four groups.

Table (1): SF-BC distances between four groups.

\begin{tabular}{lccccc} 
& \multicolumn{5}{c}{ SF-BC groups } \\
\cline { 2 - 6 } Groups & No. & Mean & SD & F & Sig. \\
\hline CG & 50 & 9.19 & 1.47 & & \\
SDE & 50 & 7.13 & 1.81 & & \\
MDE & 50 & 5.9 & 1.45 & & \\
CE & 50 & 4.74 & 1.18 & &
\end{tabular}

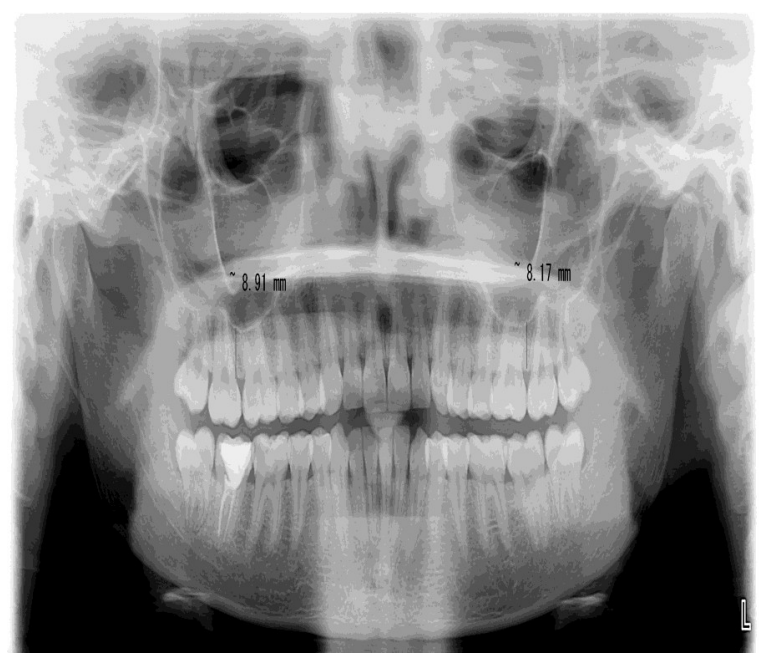

Figure 1: (SF-BC) measurement of first $\operatorname{group}(\mathrm{CG})$.

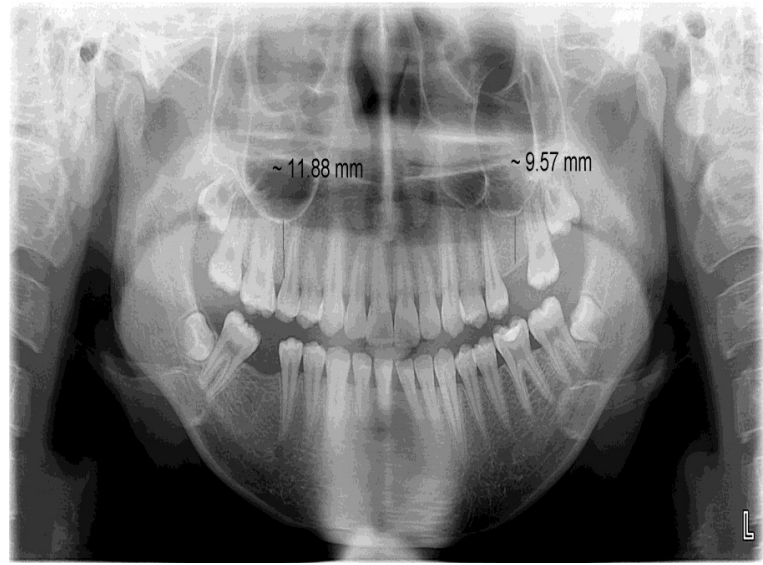

Figure 2: (SF-BC) measurement of second group (SDE).

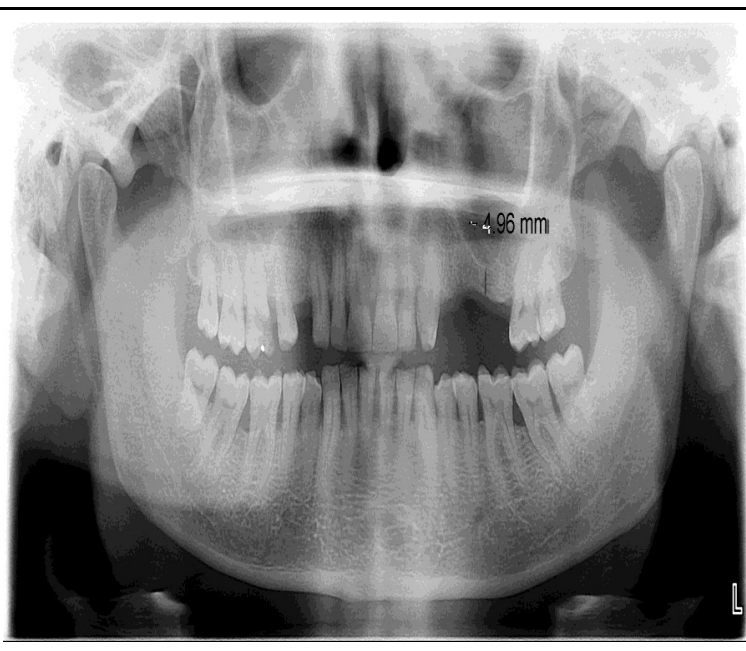

Figure 3: (SF-BC) measurement of Third group (MDE).

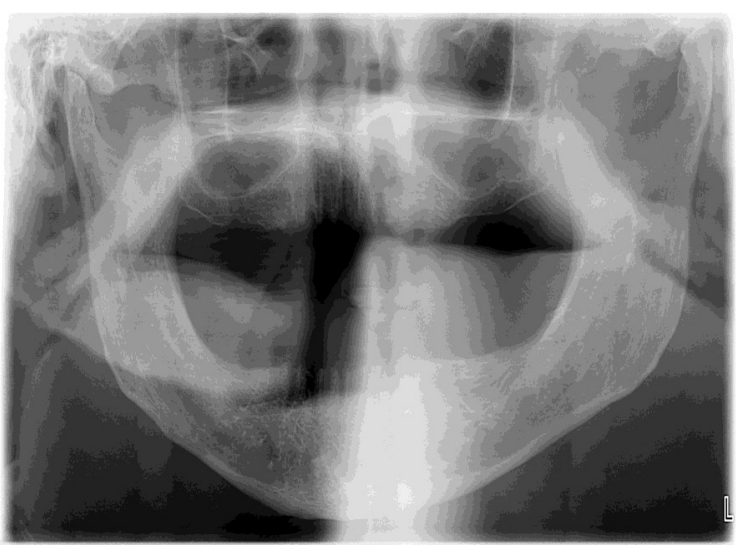

Figure 4: (SF-BC) measurement of fourth group (CE).

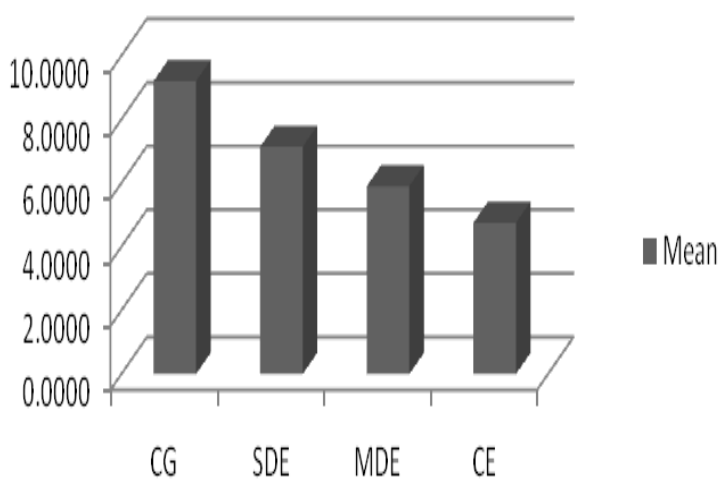

Figure 5: Comparing the means of (SF$B C)$ between four groups (CG,SDE,MDE, and CE).

SF-BC location:

SF-BC location In the male: 
The location of deepest point of SF in relation to nearest interdental $B C$ was found to be located between second. Premolar and first molar in $10 \%$ of the cases. The highest percentage was located between first and second molars which was $86 \%$ of the cases, and the Lowest percentage was located between second and third molars $4 \%$ of the cases Figure (6).

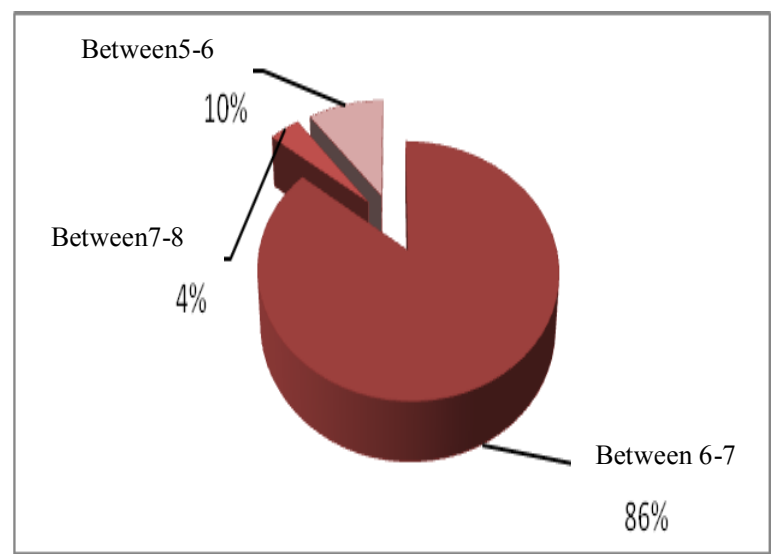

Figure 6: Showing the percentage of deepest point location SF-BC in male.

SF-BC location In the female: The location of deepest point of SF in relation to nearest interdental BC was found to be located between second premolar and first molar in $18 \%$ of the cases. The highest percentage was located between first and second molars which was $74 \%$ of the cases, and the Lowest

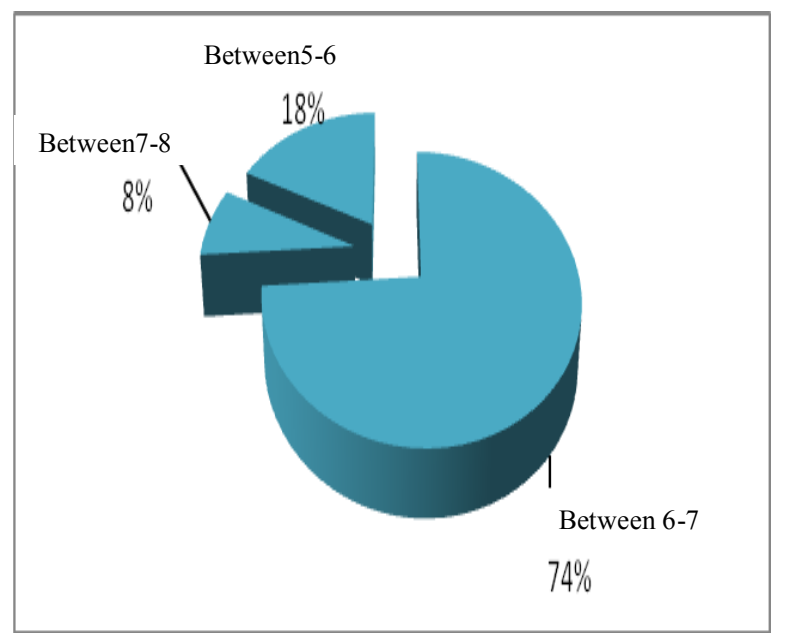

percentage was located between second and third molars $8 \%$ of the cases Figure (7).

Figure 7: Showing the percentage of deepest point location SF-BC in female.

\section{Discussion}

This retrospective cross-sectional study is the first study in Erbil city to correlate maxillary sinus floor to maxillary teeth and maxillary alveolar bone crest on digital panoramic radiography It was found out in the present study after extraction, the socket walls undergo internal and external turnover, resulting in crestal bone loss as well as horizontal reduction. Buccolingual loss overall exceeds that in the vertical direction,according to the means of all groups. There is highly significance difference $(P<0.001)$ between first, third and fourth groups while there is significance difference $(P<0.005)$ between first group and second group. The area of concern is premolar and molar area and other areas of the arch did not affect specifically the distance of sinus floor to the alveolar ridge area. This high difference may be due to extraction of upper posterior teeth and bone resorption after extraction of teeth leading to decreasing vertical dimension of alveolar ridge consequently, the maxillary sinus floor will be nearer to the alveolar bone edge. The difference in the means between second group, third group, and fourth group after dental extraction, in second group bone resorption less than in the third and fourth groups because in the second group the adjacent teeth to the extracted tooth were remain for this reason the bone resorption less than other two groups(third group and fourth group). The distance from deepest point of maxillary sinus floor to the alveolar ridge in fourth group (CE) cases reduced by approximately half in comparison with first group (CG) dentolous cases $((4.5) \mathrm{mm}$ in males and (4.99) $\mathrm{mm}$ in females). So maxillary sinus floor must be considered important during planning implant procedure for edentulous maxillae. Maxillary sinus floor (convexity) was nearer to maxillary teeth in area of maxillary first and second molar, then in area of second premolar. The 'location of deepest point (convexity) of maxillary sinus in relation to teeth was symmetrical in both 
males and females in high percent (83.5\%) of cases. For the total sample, there was no significance association between deepest point of SF-BC location with sex.

\section{References}

1. Ritter FN. The paranasal sinuses-anatomy and surgical technique. $2^{\text {nd }}$ ed. , St.Eouis: CV Mosby. 1978 ; 6-16.

2. SchovSR. Odontogenic diseases of the maxillary sinus. In: Peterson, LJ, Ellis, E, Hupp, JR, Tucker, MR. Contemporary oral and maxillofacial surgery, $2^{\text {nd }}$ ed.: Mosby, Year Book, Inc. 1993: 465-82.

3. Misch CE. Contemporary implant dentistry. $2^{\text {nd }} e d$, St. Louis: CV Mosby.1999:76-194.

4. Rothman SLG. Dental applications of computerized tomography: Surgical planning for implant placement. Illinois, Quintessence Books.1998; 109-113.

5. Bergeron RT, Osborn AG, Som PM, editors.: Head and Neck Imaging; St. Louis. Mosby. 1984.

6. Harring IJ, Jansen L. Dental Radiography-principles and techniques. $2^{n}$ ed. 2000; 24: 385-387. 23: 370.

7. Topazian RG, Golberg MH, Hupp JR. Oral and Maxillofacial infections. $4^{\text {th }}$ ed. Philadelphia, WB Saunders. 2002; 2:46-57.

8. Perez CA, Farman AG. Diagnostic radiology of maxillary sinus defects. Oral Surg Oral Med Oral Pathol. 1988; 68: 507-12.

9. Perella A, Sara SR, Marcelo GC. Quantitative analysis of maxillary sinus using computed tomography. J Appl. Oral Sci.2003; 11(3).

10. Kim HJ, Yoon HR, Kim KD, Kang MK, Kwak HH, Park HD, Han SH,Park CS. Personal-computerbased three-dimensional reconstruction and simulation of maxillary sinus. SurgRadiol Anat. 2002; 24: 393-399

11. Kwak HH, Park HD, Yoom HR, Kong MK,. Koh KS, Kirn: HJ. Topographic anatomy of the inferior wall of the maxillary sinus in Koreans. Int. J. Oral Maxillofac. Surg. 2004; 33: 382-388. 